\title{
TRANSFoRm Query Workbench
}

Theodoros N. Arvanitis ${ }^{1 *}$, Wolfgang Kuchinke ${ }^{2}$

From 1st Clinical Research Informatics (CRI) Solutions Day

Duesseldorf, Germany. 26-27 May 2014

\section{Characterisation}

Tool, open source, data management, research query generation, database research.

\section{Description}

The Query Workbench is a tool of the TRANSFoRm project to support clinical studies and database research.
It provides an interface to author, store and deploy queries of clinical data to identify potential subjects for clinical studies and can thus support the clinical study feasibility evaluation. The development of the Query Workbench system is driven by the TRANSFoRm Clinical Research Information Model (CRIM) [1], enabling the creation of a semantically aware software tool for

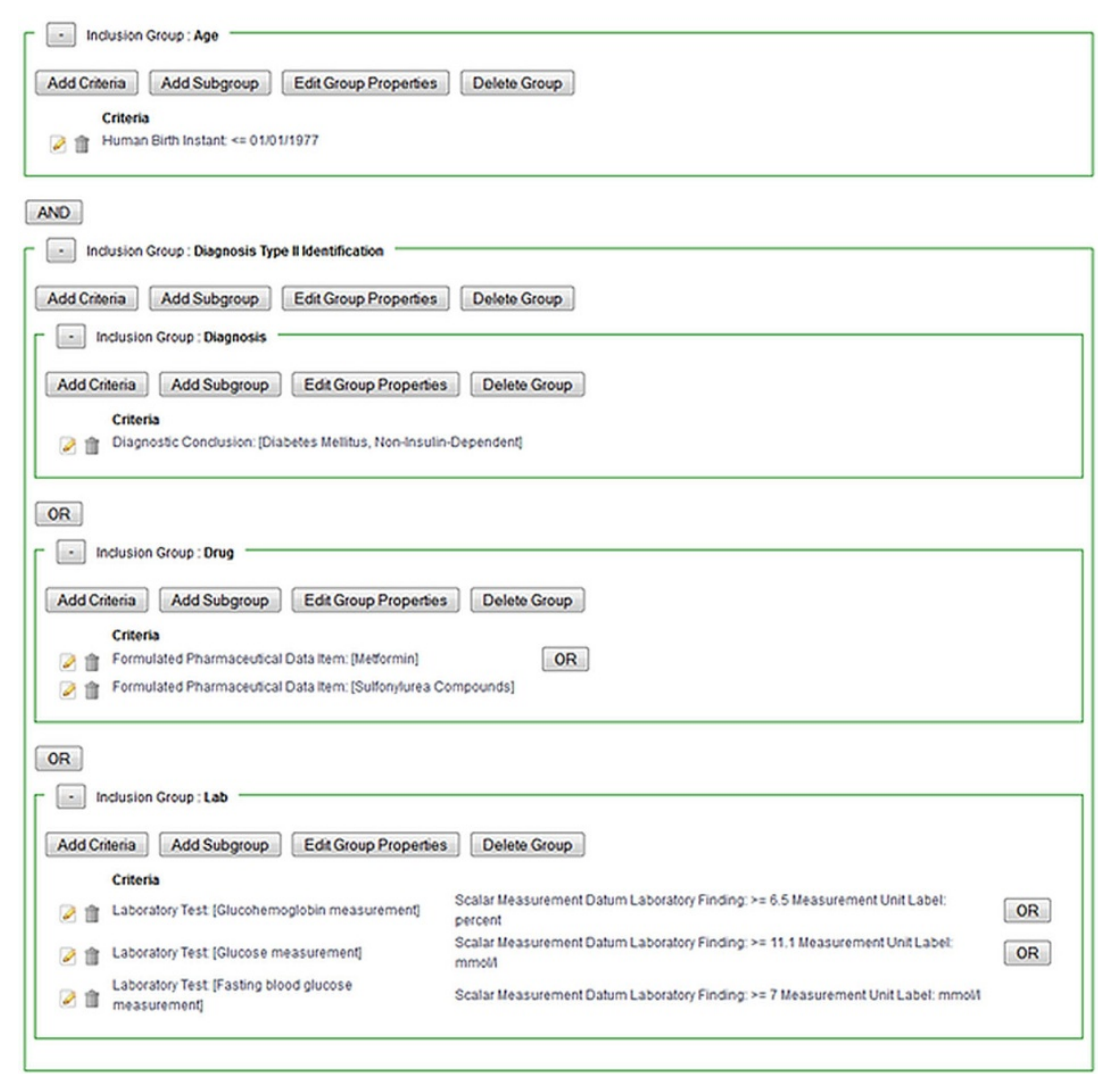

Figure 1 Query Workbench user interface. Display of definition of eligibility criteria.

* Correspondence: T.Arvanitis@warwick.ac.uk

'University of Warwick, Coventry, CV4 7AL, UK

Full list of author information is available at the end of the article

(c) 2015 Arvanitis and Kuchinke; licensee BioMed Central Ltd. This is an Open Access article distributed under the terms of the Creative 
easy authoring of distributed searches to EHR (electronic health record) and other clinical data sources (e.g. primary care databases). The use of the TRANSFoRm terminology services, in conjunction with the Clinical Data Integration Model (CDIM) [2] allows the capturing of eligibility criteria in a computable representation, based on the CDIM ontology, so the criteria can be translated into executable query statements at the individual EHR data source (Figure 1). In this way the workbench is able to automatically identify "prevalent cases" for research purposes; the queries report back counts of eligible subjects in the EHRs or the corresponding databases. Results can be broken down by eligibility criteria to give the most detailed information about the distribution of patients and to enable users to update study protocols accordingly. Flagging the subjects for recruitment and obtaining consent is done by the local clinical care team, in full compliance with data protection legislation and best practices.

Integration with other TRANSFoRm components, such as the security framework, has provided a successful working functionality of the query formulation tool within the TRANSFoRm distributed infrastructure. In this way, users can work together on study and protocol design.

In summary, the Query Workbench supports the creation and conduct of Europe-wide studies, and supports searches in multiple heterogeneous data sources, such as GPRD and NIVEL primary care databases, without the need of processing the data into a common format.

\section{Status of development}

Query Workbench is used and evaluated with different databases (e.g. NIVEL); employed in a Diabetes cohort study use case.

\section{Users}

Investigators to improve clinical study feasibility and recruitment rate; researchers interested in epidemiologi$\mathrm{cal}$ and database research.

\section{Link}

http://www.transformproject.eu/

\footnotetext{
Authors' details

${ }^{1}$ University of Warwick, Coventry, CV4 7AL, UK. ${ }^{2}$ Heinrich-Heine University Düsseldorf, University Hospital, 40225 Düsseldorf, Germany.

Published: 22 May 2015

\section{References}

1. Kuchinke W, Karakoyun T, Ohmann C, Arvanitis TN, Taweel A, Delaney BC, Speedie SM: Extension of the primary care research object model (PCROM) as clinical research information model (CRIM) for the learning healthcare system. BMC Med Inform Decis Mak 2014, 14(1):118-128.
}

2. Ethier JF, Dameron O, Curcin V, McGilchrist MM, Verheij RA, Arvanitis TN, Taweel A, Delaney BC, Burgun A: A unified structural/terminological interoperability framework based on LexEVS: application to TRANSFoRm. J Am Med Inform Assoc 2013, 20(5):986-994.

doi:10.1186/2043-9113-5-S1-S16

Cite this article as: Arvanitis and Kuchinke: TRANSFoRm Query

Workbench. Journal of Clinical Bioinformatics 2015 5(Suppl 1):S16.

\section{Submit your next manuscript to BioMed Central} and take full advantage of:

- Convenient online submission

- Thorough peer review

- No space constraints or color figure charges

- Immediate publication on acceptance

- Inclusion in PubMed, CAS, Scopus and Google Scholar

- Research which is freely available for redistribution 